\title{
Cottbus: Traffic Signal Simulation
}

\author{
Joschka Bischoff and Dominik Grether
}

The Cottbus (Germany) scenario is used for traffic light simulation (see Chapter 12). It is explained by Grether (2014, pp. 87); this chapter briefly reviews the main points. The scenario data is generally available to the public, and can be found from http://matsim.org/datasets.

The network was derived from OSM data in summer 2010 (Bischoff, 2010), and covers all streets within the city boundaries, as well as main roads in the surrounding Spree-Neiße administrative district. It is designed as a $100 \%$ sample. The population is based on the German federal employment agency commuter statistics for both Cottbus and Spree-Neiße (Wiethölter et al., 2010). As such, the population has only home-work-home plans spread over the usual commuting times, resulting in two peaks, including 33479 agents traveling exclusively by car. The scenario is generally not very busy; the area does not usually have major congestion issues.

Figure 66.1(a) shows the network over the "Corine Land Cover" landuse (European Environment Agency, 2011), provided by European Environmental Agency. Woods and agricultural areas are depicted; most of the region is agricultural use area. Virtual persons in MATSim need a geographic coordinate for their activities. If this coordinate is drawn randomly (solely based on municipality borders), home and work activity locations are uniformly distributed over the area, i.e., most of them in woods and fields. Thus, activity locations are drawn randomly in combination with land use data. The coordinate must be in the municipality area and for home activity, it must be located in urban fabric areas; for work locations, industrial or commercial areas are also allowed. The resulting home activity locations are shown in Figure 66.1(b).

The scenario contains data for 22 traffic signals within the city center, based on the city's 2009 signal plans; junction layout is also modeled in detail. Fixed-time control data is taken from Köhler and Strehler (2010). Due to higher transport network resolution, several originally recorded fixedtime control schedules are invalid and were removed; data for 22 junctions is available. Figure 66.2 shows their transport network location.

Public transit, not part of the original scenario, is available based on 2011 schedules, although it is not currently used. 


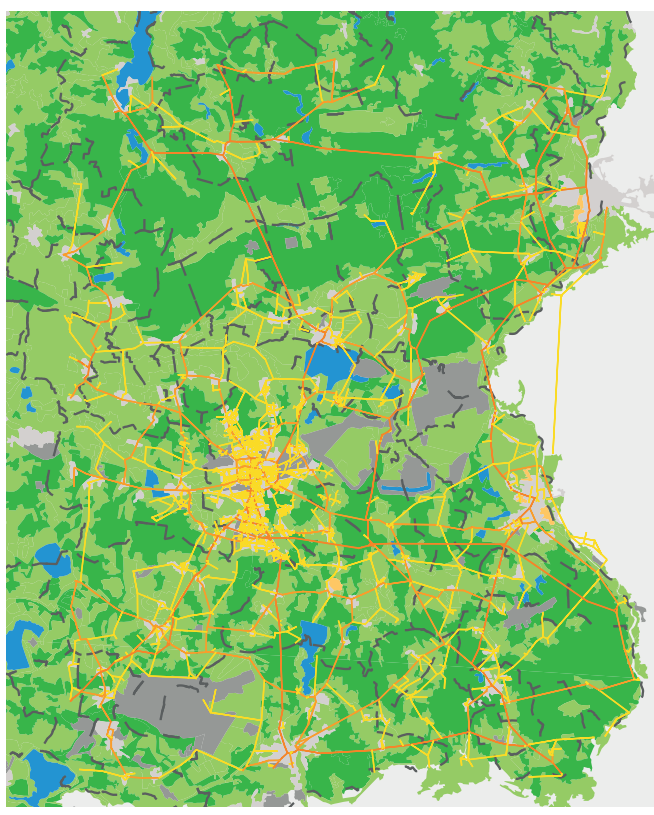

(a) Cottbus network and municipality borders.

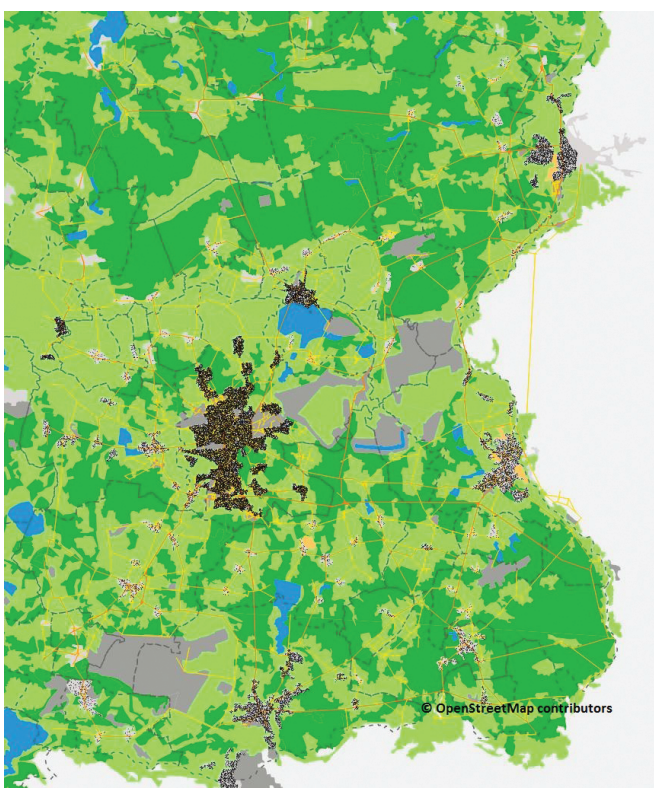

(b) Synthetic population for the Cottbus scenario, geospatial location of home activities.

Figure 66.1: Cottbus scenario: Network and population.

Source: Grether (2014)

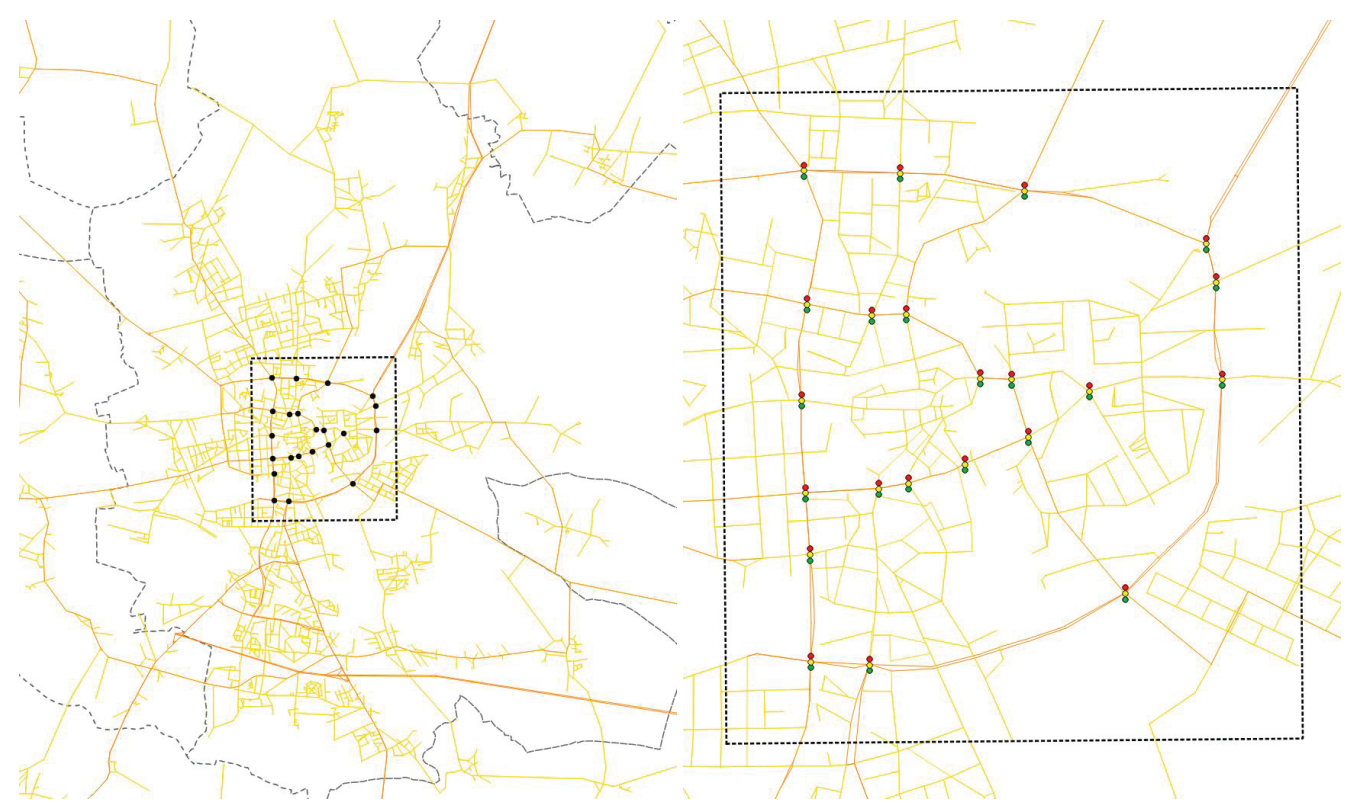

(a) Location within city of Cottbus.

(b) Signalized area in detail.

Figure 66.2: Cottbus scenario: Network, area with traffic signals within the city of Cottbus. Source: Grether (2014) 